Ann. Sci. For., 1986, 43 (2), 179-188

\title{
Importance de la teneur en eau du pollen pour la réalisation de croisements contrôlés chez le Douglas
}

\author{
Ewa MELLEROWICZ * et M. BONNET-MASIMBERT \\ INRA, Centre de Recherches d'Orléans \\ Station d'Amélioration des Arbres forestiers, Ardon, F 45160 Olivet
}

\section{Résumé}

Cette étude visait à vérifier si, comme démontré pour la germination in vitro, l'influence de l'état d'hydratation du pollen de Douglas se manifestait aussi in vivo. Les pollinisations contrôlées sont effectuées sur les inflorescences femelles de 24 arbres soit avec du pollen conservé à $-1{ }^{\circ} \mathrm{C}$ pendant 1 an avec une teneur en eau de 4 ou $10 \mathrm{p}$. 100 , soit avec du pollen de l'année séché à 4 ou 10 p. 100. L'efficacité de ce pollen, considéré comme sec, est comparée à celle des mêmes lots réhydratés à 14,30 ou 40 p. 100 . Une pourriture importante a affecté les cônes, indépendamment des traitements. Elle est cependant plus élevée lors d'utilisation de pollen à 30 p. 100 ou 40 p. 100 . En fait cette réhydratation n'influe ni sur le développement des cônes ni sur le nombre de graines plcines formées, alors que son effet favorable sur la germination in vitro a été confirmé. Les mécanismes naturels précédant la fécondation chez le Douglas permettent sans doute une bonnc réhydratation lors du séjour du pollen dans le canal micropylaire. D'un point de vue pratique, il n'y a donc pas intérêt à réhydrater le pollen de Douglas avant son utilisation en croisement. La discussion porte sur l'impossibilité de généraliser de tels résultats à l'ensemble des espèces forestières.

Mots clés : Pollen, hydratation, croisements contrôlés, Pseudotsuga menziesii.

\section{Introduction}

Dans un précédent travail, Charpentier \& Bonnet-Masimbert (1983), ont constaté que la germination in vitro du pollen de Douglas (Pseudotsuga menziesii (Mirb)) déshydraté est nettement améliorée par une réhydratation ménagée préalable à la mise en germination. On peut se demander si un phénomène analogue se produit dans le cas de la germination in vivo, lors de croisements.

Une augmentation du taux de fécondation, donc vraisemblablement du taux de germination in vivo, à la suite d'une pollinisation avec du pollen réhydraté a été observée chez Gladiolus (Pfeiffer, 1939), Dieffenbachia maculata (HENNY, 1980)

(*) Adresse actuelle : Department of Biology, University of New Brunswick, Bag Service $N^{*} 45111$, Fredericton, N.B. Canada, E3B 6E1. 
et aussi, bien qu'indirectement, chez Armeria maritima (EISIKowitch \& Woodelk, 1975). A notre connaissance, il n'existe pas d'information du même type chez des gymnospermes.

Dans cet essai, nous avons voulu vérifier si, en réhydratant le pollen avant de l'utiliser pour les croisements, nous pouvions améliorer le rendement en graines chez le Douglas.

\section{Matériel et méthode}

\subsection{Préparation du pollen}

Fin mars 1982, nous avons effectué en serre des pollinisations sur 24 arbres élevés en conteneurs appartenant à 21 clones. Le pollen provenait de 3 pères notés 1 , 2, 3, récoltés en 1981 (pollen conservé depuis à -1 "C) et de 3 pères notés 4,5 et 6 récoltés peu avant la campagne de croisements (pollen conservé à +4 "C pendant les quelques jours qui précédaient les croisements).

Le pollen des pères 1,2 et 3 a été conservé à deux différents nivcaux de teneur en eau (T.E.) : 4 p. 100 et 10 p. 100 (exprimés par rapport au poids frais). Habituellement les utilisateurs de pollen de Douglas le conservent à une T.E. de 8 à 10 p. 100 à 4 "C ou $-15^{\circ} \mathrm{C}$ (Ching \& Ching, 1976). Pour les croisements ce pollen a été utilisé soit sec (S) c'est-à-dire tel qu'il était en sortie de conservation, soit après $16 \mathrm{~h}$ de réhydratation en atmosphère à 100 p. 100 d'humidité relative à la température du laboratoire, conditions considérées comme optimales pour les tests in vitro (CraRPEnTIER \& Bonnet-Masimber'T, 1983). Dans ce dernier cas la T.E. a été portée aux environs de 40 p. 100.

Le pollen des arbres 4,5 et 6 a été déshydraté jusqu'à deux niveaux de T.E. : 4 p. 100 et 7 p. 100 et utilisé soit dans cet état, considéré comme sec (S), soit après 80 minutes de réhydratation $(r)$, ce qui a porté sa T.E. à environ 14 p. 100, soit après réhydratation de $16 \mathrm{~h}(\mathrm{R})$, ce qui l'a portée aux environs de $30 \mathrm{p} .100$.

\subsection{Germination in vitro}

Nous avons employé la technique précédemment décrite (CHARPENTIER \& BonnetMAsimberT, 1983) mais sans autoclavage du milieu puisque l'adjonction de chloramphenicol et de nystatine $(15 \mathrm{mg} / \mathrm{l}$ de chaque) empêche suffisamment le développement de microorganismes sans affecter l'allongement des grains de pollen. Ceci a été confirmé par des essais complémentaires avec des concentrations allant jusqu'à $90 \mathrm{mg} / 1$ de ces antibiotiques (Mellerowicz \& Bonnet-Masimbert, 1983).

\subsection{Technique de croisement}

Avant la campagne de croisements, les arbres ont été placés en chambre froide $\grave{a}+4$ " $\mathrm{C}$ pendant 1 à 2 semaines pour freiner le développement des bourgeons femelles jusqu'à ce que nous ayons pu récolter du pollen frais. Ils ont ensuite été transférés en serre. 
Les rameaux portant des bourgeons femelles ont été ensachés quelques jours avant leur débourrement. Nous avons utilisé des tubes de $6 \mathrm{~cm}$ de diamètre en celluloïde, fermés aux deux extrémités par des bouchons en mousse de polyuréthane de forte densité. Le bouchon de l'extrémité distale était écarté lors de la pollinisation réalisée à l'aide d'un pinceau.

Pour permettre une bonne interprétation des résultats, une seule pollinisation par inflorescence femelle a été effectuće, à un même stade de développement pour toutes les inflorescences, stade obtenı un à deux jours après le débourrement floral. Ce stade est considéré comme le plus réceptif (Owens et al., 1981).

Dans la mesure du possible, un même pollen (même père, même T.E. de départ) a été appliqué, d'une part à l'état sec, d'autre part à l'état réhydraté, sur un même clone, sur des inflorescences situées sur des rameaux d'un même verticille. Pour chaque modalité ainsi définie il y a au minimum deux inflorescences femelles. plus généralement présentes dans le même tube.

\subsection{Nature des observations}

Trois à quatre semaines après pollinisation, les arbres ont été transférés en pépi. nière. Tous les mois nous avons fait des observations sur l'état de développement des cônes : développement normal ou avortement, attaque de champignons ou d'insectes...

Au début de septembre, les cônes ont été récoltés et mesurés (longueur, diamètre). On en a extrait manuellement toutes les graines en distinguant 5 catégories :

1. Graines non développées : elles proviennent surtout de l'extrémité supéricure des cônes.

2. Graines vides : graines séparćes par tri densimétrique à l'éther de pétrole, puis coupées pour en distinguer le type.

3. Graines parasitées : graines séparées par tri densimétrique à l'éther de pétrole, puis coupées pour en distinguer le type.

4. Graines sèches : graines dont l'endosperme ne remplit pas entièrement la cavité. Elles flottent avec les graines parasitées et vides et en sont séparées lors de la coupe.

5. Graines pleines normales.

Dans la suite du texte nous désignerons par grosses graines celles qui appartiennent à l'ensemble des catégories 2, 3, 4 et 5 et par graines pleines celles des catégories 3,4 et 5 .

Les résultats ont été analysés statistiquement soit par le test de $\chi^{2}$, soit par l'analyse de variance multifactorielle.

\section{Résultats}

Si l'on s'intéresse d'abord aux résultats in vitro, on constate que la germination du pollen a été améliorée par la réhydratation pour tous les lots de 1981 et pour le 
pollen à 4 p. 100 de T.E. de 1982 (tabl. 1). Le pollen de 1982 à 7 p. 100 de T.E. a très bien germé sans réhydratation. On a observé une grande variation du comportement en fonction de l'arbre-père.

\section{TABLeau 1}

Influence de la réhydratation sur la germination in vitro de pollen conservé une année à $-1{ }^{\circ} \mathrm{C}$ et de pollen frais.

Influence of rehydration on in vitro germination of pollen stored for one year at -1 "C and fresh pollen.

\begin{tabular}{|c|c|c|c|c|c|c|c|c|c|c|}
\hline \multirow{3}{*}{ Arbre } & \multicolumn{4}{|c|}{$\begin{array}{c}\text { Germination du pollen } 1981 \\
\text { (conservé) } \\
\text { Teneur en eau }\end{array}$} & \multirow{3}{*}{ Arbre } & \multicolumn{5}{|c|}{$\begin{array}{l}\text { Germination du pollen } 1982 \\
\text { (frais) } \\
\text { Teneur en eau }\end{array}$} \\
\hline & \multicolumn{2}{|c|}{$4 \%$} & \multicolumn{2}{|c|}{$10 \%$} & & \multicolumn{2}{|c|}{$4 \%$} & \multicolumn{3}{|c|}{$7 \%$} \\
\hline & $\mathrm{S}$ & $\mathbf{R}$ & S & $\mathbf{R}$ & & $S$ & $\mathbf{R}$ & $\mathrm{S}$ & $\mathrm{r}$ & $\mathbf{R}$ \\
\hline 1 & $0^{*}$ & $35 *$ & 26 & 68 & 4 & 82 & 96 & 97 & 95 & 94 \\
\hline 2 & - & - & 28,5 & 53,5 & 5 & 5 & 89 & 95 & 96 & 96 \\
\hline 3 & & & 62,5 & 70,5 & 6 & 26 & 98 & 89 & 92 & 93 \\
\hline Moyennes & & & 39 & 64 & $\begin{array}{c}\text { Moyen- } \\
\text { nes }\end{array}$ & 37,7 & 94,3 & 93,7 & 94,3 & 94,3 \\
\hline
\end{tabular}

$\because$ Germination sans nystatine ni chloramphénicol. Les résultats des tests avec ces substances ont donné une germination nulle sans que nous en ayons déterminé la cause.

L.es pourcentages sont calculés sur une seule lame (niveau de confiance $5 \mathrm{p}, 100$ ).

$\mathrm{S}=$ Pollen sec $; \mathrm{r}=$ Pollen réhydraté $80 \mathrm{mn} ; \mathrm{R}=$ Pollen réhydraté $16 \mathrm{~h}$.

\section{Tableau 2}

Pourcentages de cônes développés en fonction de l'état du pollen utilisé pour les pollinisations.

Les moyennes pour le pollen conservé (1981) portent sur 3 familles.

Pour le pollen frais (1982), elles portent sur 13 familles.

Percentage of cones reaching maturity as a function of the type of pollen used in pollinization.

The data represent means of 3 families for stored pollen (1981), and 13 families for fresh pollen (1982).

\begin{tabular}{|c|c|c|c|c|}
\hline \multirow{3}{*}{$\begin{array}{l}\text { Année de récolte } \\
\text { Teneur en eau } \\
\text { du pollen sec }\end{array}$} & \multicolumn{4}{|c|}{ Pourcentage de cônes développés } \\
\hline & \multicolumn{2}{|c|}{1981} & \multicolumn{2}{|c|}{1982} \\
\hline & $4 \%$ & $10 \%$ & $4 \%$ & $7 \%$ \\
\hline Etat d'hydratation & & & & \\
\hline$s \ldots \ldots \ldots \ldots$ & $50 \%$ & $54 \%$ & $50 \%$ & $50 \%$ \\
\hline$[\ldots \ldots \ldots \ldots$ & - & - & - & $56 \%$ \\
\hline R & $0 \%$ & $40 \%$ & $35 \%$ & $37 \%$ \\
\hline
\end{tabular}

$\mathrm{S}=$ Pollen sec $; \mathrm{r}=$ Pollen réhydraté $80 \mathrm{mn} ; \mathrm{R}=$ Pollen réhydraté $16 \mathrm{~h}$. 
Si l'on s'intéresse maintenant aux résultats in vivo, on constate que, un mois après pollinisation, la moitié des cônes a pourri, vraisemblablement suite à une attaque de champignons qui a d'ailleurs affecté dans la serre plusieurs arbres, faisant partie d'autres expérimentations, y compris lorsqu'un autre type d'ensachage était utilisé. La pourriture des cônes tient essentiellement à un facteur arbre-mère. En effet, certaines mères ont vu tous leurs cônes avorter et d'autres mères ont vu tous leurs cônes se développer et ceci, indépendamment du pollen appliqué. Cependant, les inflorescences femelles pollinisées par le pollen réhydraté pendant 16 heures ont pourri plus souvent que celles pollinisées par le pollen sec ou réhydraté seulement pendant 80 minutes (tabl. 3). Les différences ne sont toutefois pas significatives, probablement du fait de la variabilité de réaction des arbres-mères.

Les dimensions des cônes ne dépendent pas de la réhydratation du pollen. Elles ne sont donc pas rapportées ici. Elles sont uniquement liées à l'arbre-mère et à la situation dans la cime, les cônes situés plus haut étant plus larges et plus longs que ceux situés plus bas.

\section{TABleau 3}

Moyennes du nombre de graines par cône et des pourcentages de graines obtenues par pollinisation avec du pollen de 1981 sec ou réhydraté.

L'analyse porte sur 74 cônes, appartenant à 4 familles, pollinisés uniquement avec du pollen conservé à une T.E. de 10 p. 100.

Mean number of seeds per cone and percent filled seeds resulting from pollinization with 1981 pollen, dry and rehydrated.

Seventy-four cones from four families pollinated with pollen stored at 10 p. 100 moisture content were analyzed.

\begin{tabular}{|c|c|c|c|}
\hline \multirow[b]{2}{*}{ Variable analysée } & \multicolumn{2}{|c|}{ Cônes pollinisés par } & \multirow{2}{*}{$\begin{array}{l}\text { Signification } \\
\text { des différences } \\
\text { (seuil } 1 \% \text { ) }\end{array}$} \\
\hline & $\begin{array}{l}\text { Pollen sec } \\
\qquad S\end{array}$ & $\begin{array}{c}\text { Pollen réhydraté } \\
\mathrm{R}\end{array}$ & \\
\hline Nombre de graincs extraites .... & 37,8 & 44,6 & ** \\
\hline $\begin{array}{c}\text { Nombre de graines non dévelop- } \\
\text { pées } \ldots \ldots \ldots \ldots \ldots \ldots \ldots\end{array}$ & 10,3 & 11,8 & NS \\
\hline Nombre de graines parasitées ... & 0,286 & 0,941 & ** \\
\hline Nombre de grosses graines .. & 27,5 & 32,8 & $* *$ \\
\hline Nombre de graines pleines .... & 4,78 & 4,79 & NS \\
\hline $\begin{array}{l}\% \text { de graines pleines par rapport } \\
\text { aux extraites } \ldots \ldots \ldots \ldots \ldots\end{array}$ & 13,4 & 11,8 & NS \\
\hline $\begin{array}{r}\% \text { de graines pleines par rapport } \\
\text { aux grosses graines } \ldots . . . .\end{array}$ & 18,6 & 16,4 & NS \\
\hline
\end{tabular}

: Différences significatives à 1 p. 100 . 
Les pourcentages de graines pleines ont été faibles : 12,6 p. 100 en moyenne pour les pollinisations avec du pollen eonservé et $22,8 \mathrm{p}$. 100 avec du pollen frais. Cependant, les cônes des mêmes arbres, qui nont pas été ensachés, contenaient encoré moins de graines pleines alors que le pollen libre ne leur avait vraisemblablement pas manqué. Le résultat n’a pas été influencé par la réhydratation du pollen (Tabl. 3 et 4). L'analyse des graines obtenues avec le pollen de 1981 a révélé quelques résultats significatifs qui ne se sont cependant pas confirmés avec le pollen de 1982 : augmentation du nombre de graines extraites, grosses et parasitées (par Megastigmus spermotrophus-Wachtl.) lorsque le pollen a été réhydraté : augmentation du nombre de graines extraites et grosses due au développement des graines des extrémités des cônes (de catégorie 1) en graines vides, mais de dimensions normales.

\section{TABlinu 4}

Moyennes da nombre de graines par cóme ef pourcentuges de graines obtenues par pollinisation avec du pollen de 1982 à différentes teneur e'n cau.

L'analyse porte sur 44 cones appartenant ì 6 familles.

Mean number of seeds per cone and percent filled seeds

produced by cones pollinated in 1982 with pollen at different moisture content. Forty-four cones from 6 families were analyzod.

\begin{tabular}{|c|c|c|c|c|c|}
\hline$\overline{-}-\overline{-}-\overline{-}$ & 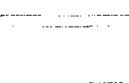 & $\overline{-\cdots}$ & Teneur en eat & du pollen & $\cdots$ \\
\hline $\begin{array}{l}\text { Variable analysée } \\
\text { (par cône) }\end{array}$ & $4 \%$ sec & $7 \%$ sec & $\begin{array}{c}7 \% \text { réhydraté } \\
\text { pendant } \\
80 \mathrm{mn}\end{array}$ & $\begin{array}{l}4 \% \text { réhydraté } \\
\text { pendant } 16 \mathrm{~h}\end{array}$ & $\begin{array}{l}7 \% \text { réhydraté } \\
\text { pendant } 16 \mathrm{~h}\end{array}$ \\
\hline $\begin{array}{c}\text { Nombre de graines extrai- } \\
\text { tes } \ldots . . . \ldots \ldots \ldots\end{array}$ & 47.8 & 51,9 & 55,0 & 47,2 & 49,0 \\
\hline $\begin{array}{c}\text { Nombre de graines non } \\
\text { développées } \ldots . . . \ldots\end{array}$ & 8,56 & 14,9 & 12,5 & 10,4 & 8,29 \\
\hline $\begin{array}{c}\text { Nombre de graines para- } \\
\text { sitées } \ldots \ldots \ldots \ldots \ldots \ldots\end{array}$ & 4,89 & 5,57 & 5,27 & 4,30 & 2,71 \\
\hline Nombre de graines sèches & 2,78 & 1,57 & 5,27 & 4,30 & 1,29 \\
\hline Nombre de grosses graines & 39.2 & 37,0 & 42,4 & 36,7 & 40,7 \\
\hline Nombre de graines pleines & 12,5 & 9,86 & 8,73 & 11,4 & 9,29 \\
\hline $\begin{array}{l}\% \text { de graines pleines par } \\
\text { rapport aux extraites .. }\end{array}$ & 24,7 & 18,8 & 22,9 & 23,4 & 25,6 \\
\hline
\end{tabular}

\section{Discussion}

De faibles taux de graines pleines et un avortement des cônes peu de temps après la pollinisation ont déjà été périodiquement observés chez le Douglas (VAN VREDENBURCH \& LA BASTIDE, 1969). Ce phénomène pourrait être lié à l'état physio- 
logique des arbres ou/et aux conditions climatiques au cours du développement des inflorescences femelles. Il semble que la pollinisation avec du pollen très humide (30-40 p. 100 T.E.) puisse avoir un effet nocif sur ce développement. Lors de la déhiscence naturelle du pollen, nous avons cependant observé assez fréquemment, de telles T.E. Dans notre expérimentation, ce résultat s'est répété systématiquement, bien qu'il n'ait pas été statistiquement confirmé. Une augmentation de l'humidité peut surtout favoriser l'infection par des champignons, ce que nous avons observé. Nous ne pouvons pas dire, cependant, si ces infections sont la cause principale de l'avortement des cônes ou bien si elles ne sont apparues qu'ultérieurement.

On peut s'interroger sur l'augmentation significative du nombre de graines extraites, duc à l'augmentation du nombre de grosses graines, dans le cas du pollen réhydraté de 1981. En fait, elles se trouvent aux extrémités des cônes et, bien que plus volumineuses, elles sont restées vides. On sait que 20 p. 100 (Owens et al.. 1981) à près de 50 p. $100(\mathrm{Ho}, 1980)$ des écailles ovulifères ne portent pas d'ovules ou seulement des ovules incomplètement développés. Elles se trouvent aux deux extrémités des cônes. L'humidité du pollen a peut être permis un certain développement des téguments internes de ces graines vaines.

Il apparaît clairement que la réhydratation du pollen n'influe pas favorablement sur l'aptitude à féconder de ce dernier, alors que cette réhydratation confirme son intérêt dans le cas de la germination in vitro. Les raisons d'une mauvaise germination in vitro du pollen déshydraté ne sont pas bien expliquées (GILISSEN, 1971 ; HesiopHarrison, 1979 ; Charpentier \& Bonnet-Masimbert, 1983). On peut penser à une désorganisation des membranes cytoplasmatiques au cours de la déshydratation, hypothèse émise pour les semences (Simon, 1978) et adoptée pour le pollen par HESLOP-HARRISON (1979). Leur réorganisation suppose une réhydratation ménagée du pollen avant de le placer dans le milieu de germination. Quand on plonge ce pollen directement dans ce milieu il peut être soit abimé par afflux incontrôlé d'eau et apparaître mort, soit reconstituer incomplètement son système de membranes, ce qui facilite son éclatement. Cela a été observé avec le pollen déshydraté d'Armeria maritima (EIsIKowitch \& Woodell, 1975) et de Douglas (nos observations, non publiées). Une difficulté de germination in vivo du pollen déshydraté a également été observée chez quelques angiospermes (PfEIfFer, 1939; Henny, 1980). En fait, la réussite de la germination dépend non seulement de l'état du pollen mais aussi de l'état des stigmates chez les angiospermes (Heslop-Harrison \& Shivanna, 1977), ou des mécanismes de la fécondation chez les gymnospermes. Chez le Douglas, ce mécanisme est assez bien connu (Ho, 1980 ; Owens et al., 1981). D'après ces auteurs, le pollen de Douglas séjourne une semaine à l'entrée du canal micropylaire, puis est transporté à l'intérieur de ce canal et ne commence à s'allonger (ensemble du grain) qu'après sept jours. La germination n'est terminée qu'après plus d'un mois. Comme le canal micropylaire est fermé, la réhydratation du pollen peut être contrôlée par l'arbre-mère. Ainsi, même le pollen très déshydraté (T.E. 4 p. 100), qui n’est pas apte à germer in vitro peut retrouver son pouvoir fécondant in vivo.

On peut s'attendre à un phénomène analogue chez le mélèze, dont le mécanisme de germination in vivo est très proche de celui du Douglas (Christiansen, 1972). Par contre, chez les pins et les sapins, le pollen germe aussitôt après la pollinisation. Dans ce cas il est possible que sa forte déshydratation puisse provoquer de faibles taux de fécondation.

Quant aux angiospermes forestiers, ils appartiennent surtout à la catégorie des plantes à stigmates secs, à l'exception de Prunus (Heslop-Harrison \& SHIvanna, 
1977). Ce genre de stigmates assure une réhydratation contrôlée du pollen (HesLopHarrison, 1979), mais le temps de réceptivité peut être le facteur limitant de cette réhydratation. Ainsi on a observé chez Gladiolıs, plante à stigmates secs, que le rendement en graines était bien meilleur, quand on réhydratait le pollen à 65 p. 100 d'humidité relative avant la pollinisation (PFElfFer, 1939). Par contre, les conditions de germination sur stigmates humides semblent être comparables, du point de vue de la réhydratation du pollen, à ce qui se passe dans un milieu de germination.

En fait, chez le Douglas, tous les effets négatifs de la pollinisation avec du pollen réhydraté se rapportent au pollen très humide (30-40 p. 100) et non au pollen réhydraté jusqu'à 14 p. 100. Pour les autres espèces, il n'est donc pas exclu que le degré optimal de réhydratation pour la pollinisation soit plus bas que pour la germination in vitro. En effet, dans la nature, l'humidité excède rarement 30 p. 100 lors de la déhiscence mais baisse sans donte rapidement pendant le transport vers les organes femelles. D'autre part, des techniques de conservation du pollen exigent une déshydratation beaucoup plus forte qu'elle n'a lieu lors de la pollinisation naturelle. Des mécanismes de fécondation autres que celui du Douglas n'y sont pas forcément adaptés. En conclusion, il nous semble qu'il sera utile d'examiner la germination in vivo du pollen en fonction de son état d'humidité chez les autres espèces forestières. Ceci sera particulièrement important pour les essences pour lesquelles des techniques de conservation du pollen basées sur une très forte déshydratation sont envisagées.

Reçu en avril 1985.

Accepté en octobre 1985.

\section{Summary \\ The importance of moisture content of pollen used in controlled crosses for Douglas-fir}

This paper concerns the effect of moisture content of Douglas-fir pollen on seed production. Receptive ovulate cones on 24 trees were pollinated under controlled conditions with pollen stored at $-1{ }^{\circ} \mathrm{C}$ for one year at 4 and $10 \mathrm{p}$. 100 moisture content, fresh pollen dried to 4 and 7 p. 100 moisture content and these same lots of pollen rehydrated to 14 , 30 and 40 p. 100 moisture content. Fungi destroyed many cones regardless of the treatments, but this damage appeared to be more pronounced with pollen at 30 and $40 \mathrm{p}$. 100 moisture content. On the whole, rehydration of pollen does not affect cone development (tabl. 2) or the number of filled seeds (tabl. 3, 4). On the other hand, the effect of moisture content on in vitro germination was once again demonstrated (tabl. 1). It is possible that after pollination natural processes permit rehydration which is necessary for fertilization. The reasons why these results cannot be generalized to other tree species are discussed. For pratical purpose, pollen of Douglas-fir has not to be rehydrated before using it for crosses.

\section{Références bibliographiques}

Charpentier J.P., Bonnet-Masimblet M., 1983. Influence d'une réhydratation préalable sur la germination in vitro du pollen de Douglas Pseudotsuga menziesii après conservation. Ann. Sci. For., 40 (3), 309-317.

Ching T.M., Ching K.M., 1976. Rapid viability test and aging study of some coniferous pollen. Can. J. For. Res., 6, 516-522. 
Christiansen H., 1972. On the development of pollen and the fertilization mechanisms of Larix and Pseudotsuga menziesii. Silvae Genetica, 21 (5), 166-174.

Eisikowitch D., Woodeil S.R.J., 1975. Some aspects of pollination ecology of Armeria maritima (Mill.) Willd., in Britain. New Phytol., 74, 307-322.

Gilissen L.J.W., 1977. The influence of relative humidity on the swelling of pollen grain in vitro. Planta, 137, 229-301.

HENNY R.J., 1980. Relative humidity affects in vivo pollen germination and sced production in Dieffenbachia maculata «Perfection». J. Am. Soc. Hort. sci., 105 (4), 546-548.

Heslop-Harrison J., 1979. An interpretation of the hydrodynamics of pollen. Am. J. Bot., 66, 737-743.

Heslop-Harrison Y., Shivanna K.R., 1977. The receptive surface of the angiosperm stigma. Ann. Bot., 41, 1233-1258.

Ho R.H., 1980. Pollination mechanism and seed production potential in Douglas fir. For. Sci., 26 (4), 522-528.

Mellerowicz E., Bonnet-Masimber't M., 1983. Pollen de Douglas, Mélèzes, Aulnes et Peupliers. Bilan des recherches conduites en 1982-1983. Doc. Stat. Amél. Arbres Forestiers. INRA, Orléans, 6/83, 51 p. + 66 p., tabl. et fig.

OWens J.N., Simpson S.J., Molder M., 1981. The pollination mechanism and the optimal time of pollination in Douglas-fir Pseudotsuga menziesii. Can. J. For. Res., 11, 36-50.

Pfeiffer N., 1939. The life of Gladiolus pollen prolonged by controlled conditions of storage. Contrib. Boyce Thompson Inst., 10, 429-440.

Simon E.W., 1978. Plant membranes under dry conditions. Pestic. Sci., 9, 169-172.

Van Vredendurch C.L.H., La Bastide J.G.A., 1969. The influence of meteorological factors on the cone crop of Douglas-fir in the Netherlands. Silvae Genetica., 18, 182-186. 